\title{
Dual Modal Ultrasound Elastography of the Sternocleidomastoid Muscle in Healthy Infants: A Prospective Study
}

\author{
Na Xu, MD ${ }^{a}$, Bei Xia, MD ${ }^{a, *}$, Wei Shi, MD ${ }^{b}$, Ningning Tang, MD ${ }^{a}$, ZhiXia Wu, MD ${ }^{a}$, Binxuan Huang, MD ${ }^{a}$, \\ Hongwei Tao, $M D^{a}$ \\ ${ }^{a}$ Department of Ultrasonography, Shenzhen Children's Hospital, Shenzhen 518026, Guangdong, China; ${ }^{b}$ Department of orthopedics, \\ Shenzhen Children's Hospital, Shenzhen 518026, Guangdong, China \\ Received August 21, 2019; revision received October 12; accepted November 6. \\ Objective: To evaluate the reliability of ultrasonographic (US) elastography of the sternocleidomastoid (SCM) muscle and to \\ define normal strain ratio and shear wave velocity (SWV) values in healthy infants. \\ Methods: Two hundred healthy infants (mean age \pm standard deviation, $1.64 \pm 1.78$ month; 113 boys and 87 girls) were included \\ in this prospective study. The thickness of bilateral SCM muscles was measured by B-mode ultrasonography, and the stiffness \\ of SCM muscles was measured in both the longitudinal and transverse sections, symmetrical and extensional supine position, \\ by using strain and shear wave elastography. The correlation between thickness and elastic values of the SCM muscle and the \\ following possible influential factors were evaluated: sex, different sides of SCM muscle, different ultrasonic sections and \\ different infant positions.
}

Results: Both sex and the side of SCM muscle did not show significant correlation with the thickness or stiffness of the SCM muscle $(P>0.05)$. The stiffness of SCM muscle in the longitudinal section was significantly greater than in the transverse section $(P<0.05)$. The measurements of the SCM muscle in the stretching position were significantly greater than those in the symmetrical position $(P<0.05)$.

Conclusion: The stiffness of SCM measured by US elastography is affected by relative positions of the infants. Therefore, the factor should be taken into account when measuring the stiffness of SCM by US elastography. US elastography can evaluate the stiffness of SCM, which is helpful for clinical diagnosis and treatment of children with torticollis.

Keywords: Neck muscle; Ultrasonography; Elastography; Infants

Advanced Ultrasound in Diagnosis and Therapy 2019;04:182-186

DOI: 10.37015/AUDT.2019.191219

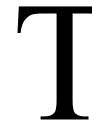
orticollis, the phenomenon of sustained abnormal neck posture, may result from various causes. Congenital muscular torticollis, neurogenic torticollis bony torticollis, and so on, can all cause torticollis [1]. However, different diseases can cause sternocleidomastoid muscle stiffness changes. Therefore, quantitative measurement of SCM stiffness is important in the treatment of sternocleidomastoid disorders [2].
It is known that changes in tissue stiffness are involved in various pathological processes, such as cancerous masses, fibrosis and calcification. A technique for objectively assessing tissue stiffness was recently made widely available with the commercial introduction of ultrasound elastography. Ultrasound elastography assesses the strain or elasticity of tissues. There are two primary methods of performing elastography:

* Corresponding author: Department of Ultrasonography, Shenzhen Children's Hospital, 7019, Yitian Road, Shenzhen, Guangdong, China

e-mail:xiabeimd@gmail.com 
strain elastography and shear-wave elastography. Strain elastography, also referred to as compression elastography, is the most commonly used method of elastography. It is based on the principle that compression of tissue produces strain. Harder tissues are less compressible and demonstrate lower strain, while softer tissues are more compressible and demonstrate higher strain $[3,4]$. Unlike strain elastography, shear wave elastography allows the determination of the actual elastic modulus of tissues. It calculates shear elastic modulus via the measurement of the velocity of a local shear wave produced by a remote mechanical source. Shearwave elastography is integrated in some commercial medical ultrasound device, thus permitting elastographic assessment complementarily to conventional ultrasound B-mode images. As it generates its own wave, the device does not require external compression from the operator and allows for real time quantitative measurements $[5,6]$. Elastography has already been used in the breast $[7,8]$, thyroid [9,10], liver [11,12], lymph nodes [13], prostate [14] and skeletal muscle $[15,16]$. Few studies have focused on the assessment of sternocleidomastoid using US elastography, and only one recent study focused specifically on congenital muscular torticollis $[2,17]$.

We studied the US elastography appearance of normal bilateral SCM muscle in healthy infant boys and girls, with different postures to determine the influential factors of the measurements and to provide a reference for evaluation of the stiffness of SCM muscle.

\section{Patients and Methods}

Two hundred healthy infants (113 boys and 87 girls, mean age $\pm \mathrm{SD}, 1.64 \pm 1.78$ months; range 13 days- 13 months) were prospectively collected from September 2017 to May 2018 in Shenzhen Children's Hospital ultrasound department. The ethical committee of Shenzhen Children's Hospital approved the study. Children with underlying abnormalities causing significant limitation of neck movement (including osteopathic torticollis, ophthalmic torticollis, neurogenic torticollis, spasmodic torticollis, and postural torticollis) were excluded from the study.

The examination was performed using Mindray's Resona7 (Mindray Medical International, China), equipped with an L14-5WU probe. US exams were performed by one sonographer in our department who was trained for more than five years in pediatric ultrasound and elastography.

All infants were examined after falling asleep. Uncooperative children received an enema using $10 \%$ chloral hydrate for sedation. The neck of the patient was symmetrically supine (Fig. 1A) and then extended to one side to maximum (Fig. 1B). The examination area was fully exposed, and the probe was transversely (Fig. 2A) and longitudinally (Fig. 2B) scanned along the SCM muscle. Local thicker gel should be used during the examination. Minimal pressure should be applied. First, to display the conventional B-mode imaging, measure the thickness of the SCM muscle in the longitudinal section and transverse section. Then turn on the shear wave mode (Fig. 2C and Fig. 2D) and the strain mode (Fig. 2G and Fig. $2 \mathrm{H}$ ). After the image is stable, freeze the image and measure the value to obtain shear wave velocity (in $\mathrm{m} / \mathrm{s}$ ), shear wave modulus (in $\mathrm{kPa}$ ) (Fig. 2E and Fig. 2F), and strain ratio (Fig. 2I and Fig. 2J) of SCM muscle. Strain ratio is the relative stiffness of the SCM muscle compared to that of the subcutaneous tissue. Three consecutive measurements were performed at the same location, and the median value was calculated for statistical analysis. The results of shear wave elastography and strain elastography are shown in Figure 2.

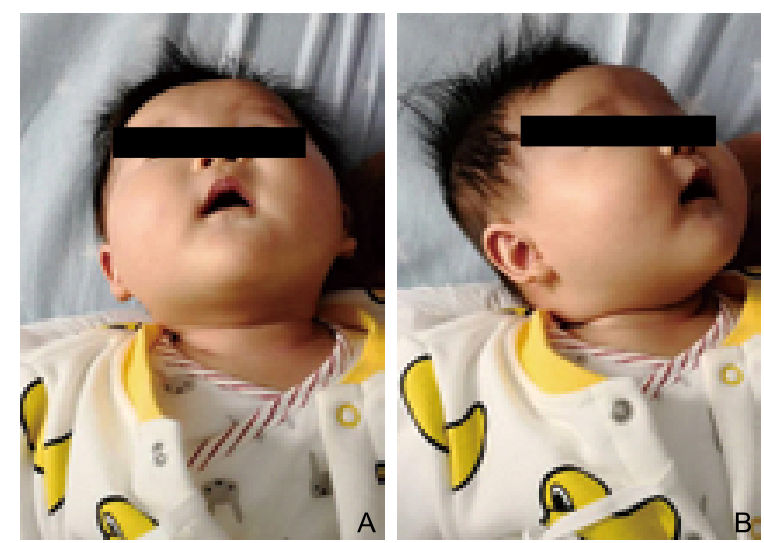

Figure 1 A 8-month-old boy with the neck symmetrically supine (A) and extended to left to maximum angle (B). The examination area was fully exposed, and the probe was transversely (A) and longitudinally (B) scanned along the SCM muscle.

In Figure 2, in the sonoelstrogram (F), the histogram indicates the stiffness of the ROI of SCM muscle; the transverse $(\mathrm{G})$ and longitudinal $(\mathrm{H})$ view of SCM muscle in strain elastography, measuring the strain ratio of SCM muscle (I) and displaying the histogram (J). In the sonoelastogram $(\mathrm{G}, \mathrm{H})$, the colors ranging from red (hard) to blue (soft) represent the relative stiffness of the tissue. In sonoelastogram (I), the upper circle (strain 1) indicates ROI of the subcutaneous tissue (reference tissue), and lower circle (strain 2) is ROI of SCM muscle. In the sonoelastogram $(\mathrm{J})$, the left histogram indicates the stiffness of ROI of the subcutaneous tissue, and the right histogram indicates the stiffness of ROI of SCM muscle.

\section{Statistical analysis}

Continuous parametric data are reported as a mean 
and standard deviation (SD). The independent sample t-test was used to compare the thickness, shear wave velocity, shear modulus, and strain ratio of the SCM muscle. All $\mathrm{P}$ values were from 2 -sided tests and results were deemed statistically significant at $P<0.05$. All statistical analyses were performed using SPSS, version 23.0 (SPSS, Inc., Chicago, IL).
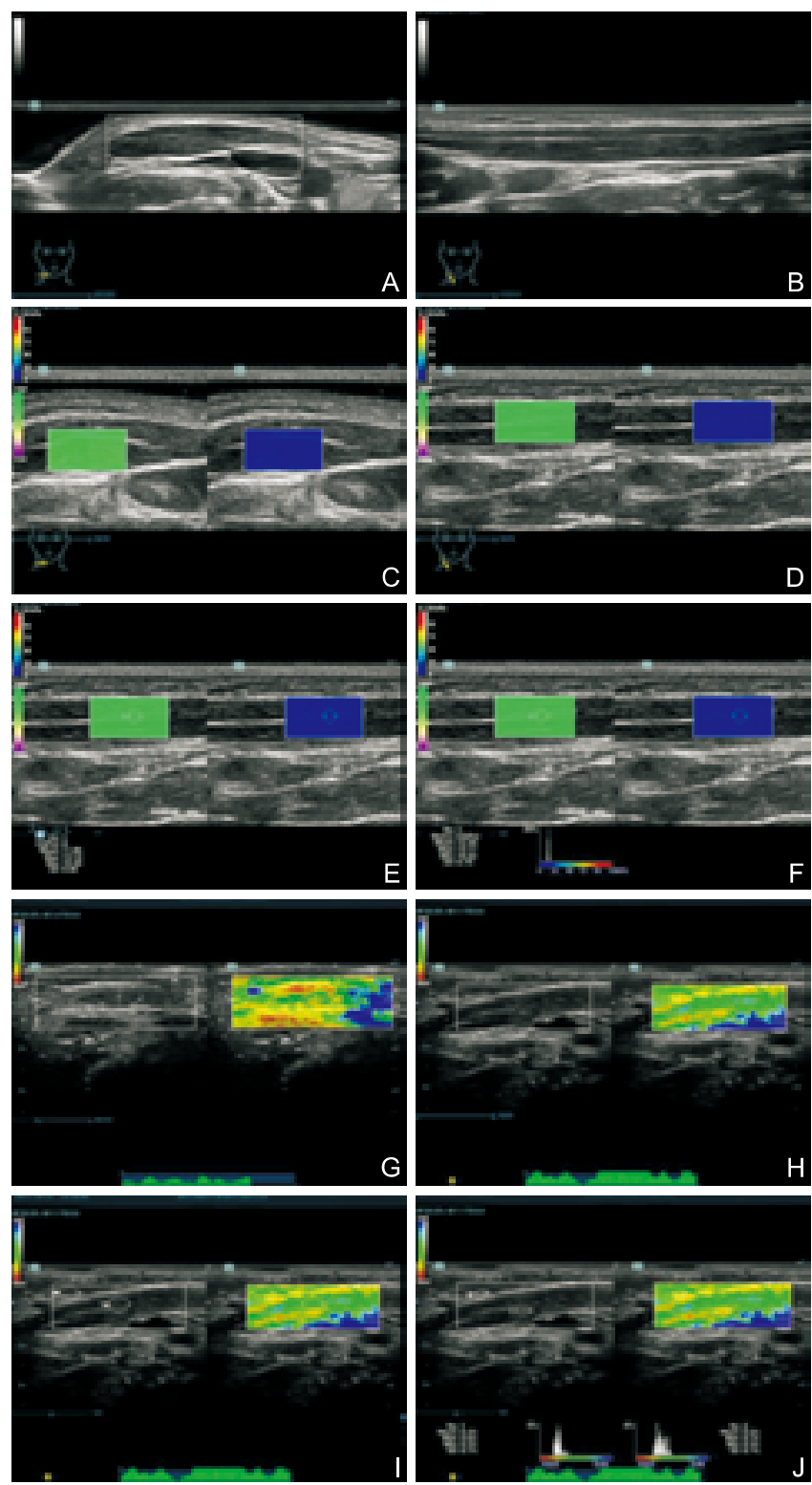

Figure 2 Transverse (A) and longitudinal (B) two-dimensional B-mode sonographic image of right SCM muscle. White arrow indicates thickness of the SCM muscle. Transverse (C) and longitudinal (D) view of SCM muscle in shear wave elastography, measuring the SCM muscle stiffness (E) and displaying the histogram (F). In sonoelastogram (E), the circle is region of interest (ROI) of the SCM muscle. There are shear wave values in the lower right.

\section{Results}

\section{Elastography parameters}

Results are summarized in Tables 1, 2, 3 and 4. In the symmetrical position of 200 infants, there was no statistical difference in the SCM muscle thickness and elastic measurements (-shear wave velocity, shear wave modulus, and strain ratio) between boys and girls $(P>$ 0.05) (Table 1).

In the symmetrical position of 200 infants, there was also no statistical difference in comparing bilateral SCM thickness and elastic measurements (-shear wave velocity, shear wave modulus, and strain ratio $)(P>0.05)$ (Table 2).

In the symmetrical position of 200 infants, the elasticity measurement (shear wave velocity, shear wave modulus) of SCM muscle in longitudinal section was significantly greater than that in the transverse section $(P$ $<0.05)$.However, there was no statistical difference in SCM strain ratio $(P>0.05)($ Table 3$)$.

In the stretching supine position of 200 infants, the shear wave measurements of the SCM muscle (shear wave velocity, shear modulus) in the transverse section and longitudinal section were significantly greater than those in the symmetrical supine position $(P<0.05)$. However, there was no significant difference in SCM muscle thickness and strain ratio $(P>0.05)$ (Table 4).

\section{Discussions}

US elastography has become a research hotspot in the field of ultrasound medicine. It is one of the new ultrasonic diagnostic techniques developed in recent years. It can evaluate the tissue stiffness in ROI. Shi et al. [18] founded that the hardness of the diseased tissue is positively correlated with the degree of tissue fibrosis. The more obvious the tissue fibrosis is, the greater the tissue hardness is. Therefore, this study used the dual modal US elastography, including strained elastography and shear wave elastography, to quantitatively analyze the stiffness of normal SCM muscle which can enable more comprehensive examination of the SCM muscle.

Skeletal muscles may exhibit different tension under different states and consequently cause different stiffness [19-21]. In the present study, 200 healthy infants were examined with SCM muscle in two different positions which included the symmetrical supine position and the extensional supine position. It was found that the shear wave velocity and shear modulus of the SCM muscle in the extensional supine position were higher than those in the symmetrical supine position. This finding was the same as reported by other studies[19-21]. Therefore, a unified posture should be taken in the stiffness measurements of SCM muscle to avoid the effect of different tension on the results. Skeletal muscle is anisotropic, so the propagation velocity of shear wave in skeletal muscle is related to the direction of muscle fiber arrangement [22]. Some researchers used the 
Table 1 Comparison of SCM muscle thickness and elastic measurement of boy and girl infants in normal group

\begin{tabular}{|c|c|c|c|c|c|c|}
\hline \multirow{2}{*}{ Item } & \multicolumn{3}{|c|}{ Short axis } & \multicolumn{3}{|c|}{ Long axis } \\
\hline & Boy & Girl & $P$ value & Boy & Girl & $P$ value \\
\hline Thickness (mm) & $3.90 \pm 0.40$ & $3.80 \pm 0.30$ & 0.121 & $3.70 \pm 0.30$ & $3.70 \pm 0.40$ & 0.140 \\
\hline Shear wave velocity $(\mathrm{m} / \mathrm{s})$ & $2.09 \pm 0.18$ & $1.97 \pm 0.21$ & 0.902 & $2.21 \pm 0.19$ & $2.12 \pm 0.19$ & 0.611 \\
\hline Shear modulus $(\mathrm{kPa})$ & $4.54 \pm 0.77$ & $4.02 \pm 0.83$ & 0.911 & $4.98 \pm 0.85$ & $4.58 \pm 0.82$ & 0.553 \\
\hline Strain ratio & $0.97 \pm 0.09$ & $1.02 \pm 0.06$ & 0.322 & $0.99 \pm 0.07$ & $0.97 \pm 0.08$ & 0.362 \\
\hline
\end{tabular}

Table 2 Comparison of left and right SCM muscle thickness and elastic measurement in normal infants

\begin{tabular}{|c|c|c|c|c|c|c|}
\hline \multirow{2}{*}{ Item } & \multicolumn{3}{|c|}{ Short axis } & \multicolumn{3}{|c|}{ Long axis } \\
\hline & Right & Left & $P$ value & Right & Left & $P$ value \\
\hline Thickness (mm) & $3.7 \pm 0.30$ & $3.80 \pm 0.60$ & 0.652 & $3.60 \pm 0.30$ & $3.70 \pm 0.5$ & 0.593 \\
\hline Shear wave velocity $(\mathrm{m} / \mathrm{s})$ & $2.03 \pm 0.20$ & $2.11 \pm 0.25$ & 0.273 & $2.23 \pm 0.22$ & $2.32 \pm 0.16$ & 0.282 \\
\hline Shear modulus $(\mathrm{kPa})$ & $4.27 \pm 0.80$ & $4.26 \pm 1.04$ & 0.811 & $4.89 \pm 0.96$ & $5.41 \pm 0.71$ & 0.492 \\
\hline Strain ratio & $1.00 \pm 0.06$ & $0.99 \pm 0.08$ & 0.754 & $0.98 \pm 0.08$ & $1.01 \pm 0.08$ & 0.731 \\
\hline
\end{tabular}

Table 3 Comparison of SCM muscle elastic measurement between short axis and long axis of normal infants

\begin{tabular}{|c|c|c|c|c|c|c|}
\hline \multirow{2}{*}{ Item } & \multicolumn{3}{|c|}{ Right } & \multicolumn{3}{|c|}{ Left } \\
\hline & Short axis & Long axis & $P$ value & Short axis & Long axis & $P$ value \\
\hline Shear wave velocity $(\mathrm{m} / \mathrm{s})$ & $2.03 \pm 0.20$ & $2.23 \pm 0.22$ & 0.030 & $2.11 \pm 0.25$ & $2.32 \pm 0.16$ & 0.031 \\
\hline Shear modulus $(\mathrm{kPa})$ & $4.27 \pm 0.80$ & $4.89 \pm 0.96$ & 0.041 & $4.26 \pm 1.04$ & $5.41 \pm 0.71$ & 0.040 \\
\hline Strain ratio & $1.00 \pm 0.06$ & $0.98 \pm 0.08$ & 0.662 & $0.99 \pm 0.08$ & $1.01 \pm 0.08$ & 0.663 \\
\hline
\end{tabular}

Table 4 Comparison of the SCM muscle thickness and elastic measurement in symmetrical and extensional supine position of normal infants

\begin{tabular}{|c|c|c|c|c|c|c|}
\hline \multirow{2}{*}{ Item } & \multicolumn{3}{|c|}{ Short axis } & \multicolumn{3}{|c|}{ Long axis } \\
\hline & Symmetric position & extension position & $P$ value & Symmetric position & extension position & $P$ value \\
\hline Thickness (mm) & $3.70 \pm 0.30$ & $3.70 \pm 0.30$ & 0.680 & $3.60 \pm 0.30$ & $3.60 \pm 0.40$ & 0.710 \\
\hline Shear wave velocity $(\mathrm{m} / \mathrm{s})$ & $2.03 \pm 0.20$ & $2.21 \pm 0.21$ & 0.039 & $2.23 \pm 0.22$ & $2.38 \pm 0.20$ & 0.036 \\
\hline Shear modulus (kPa) & $4.27 \pm 0.80$ & $4.82 \pm 0.94$ & 0.035 & $4.89 \pm 0.96$ & $5.62 \pm 0.93$ & 0.039 \\
\hline Strain ratio & $1.00 \pm 0.06$ & $1.03 \pm 1.10$ & 0.101 & $0.98 \pm 0.08$ & $1.07 \pm 0.09$ & 0.329 \\
\hline
\end{tabular}

elastic imaging technique to examine and evaluate the transverse section (the shear wave perpendicular to the muscle fiber conduction) and the longitudinal section (the shear wave parallel to the muscle fiber) of the biceps brachii muscle, gastrocnemius, rectus femoris, biceps and rectus abdominis in healthy male volunteers. It was found that shear wave propagates differently in the transverse direction and the longitudinal direction of muscle tissue. The measured values of different sections of the same muscle showed that there were significant differences in that of the short axis and the long axis of the muscle tissue [23,24]. The SCM muscle was scanned by transverse and longitudinal sections to observe the different elastic modulus. We found that the shear wave velocity and shear modulus of the longitudinal sections of the SCM muscle were higher than those of the transverse sections, which is consistent with repors in the literature [22-24]. Jiang et al. [25] examined the SCM muscle of 210 healthy adult volunteers, and found that the elastic modulus of the SCM muscle was not 
influenced by sex and age, which was consistent with the results of this study. What's more, in this study, the bilateral SCM muscles were compared, and it was found that there was no difference in the thickness, strain ratio, shear wave velocity and shear modulus of the bilateral SCM muscles.

At present, the research on the US elastography in the skeletal muscles of extremities is becoming more mature, but there are few reports about its use in SCM muscle. In this study, the factors affecting the stiffness of the SCM muscle were investigated by dual mode US elastography. Our findings indicated that the US elastic measurement of the SCM muscle is related to ultrasonic section and the degree of the neck extension, but it is not related to the sex of the children and the left and right side of the SCM muscle. These findings should be considered when applying ultrasound elastography to evaluate the stiffness of the SCM muscle.

\section{Funding}

This study was supported by the Shenzhen Health and Family Planning System scientific research project (SZFZ2017086).

\section{Conflicts of Interest}

The authors declare that there is no conflict of interest.

\section{References}

[1] Do TT. Congenital muscular torticollis: current concepts and review of treatment. Curr Opin Pediatr 2006;18:26-9.

[2] Hong SK, Song JW, Woo SB, Kim JM, Kim TE, Lee ZI. Clinical Usefulness of sonoelastography in infants with congenital muscular torticollis. Ann Rehabil Med 2016;40:28-33.

[3] Klauser AS, Peetrons P. Developments in musculoskeletal ultrasound and clinical applications. Skeletal Radiol 2010;39:1061-71.

[4] Shiina T. JSUM ultrasound elastography practice guidelines: basics and terminology. J Med Ultrason 2013;40:309-23.

[5] Franchi-Abella S, Elie C, Correas J M. Ultrasound elastography: advantages, limitations and artefacts of the different techniques from a study on a phantom. Diagn Interv Imaging 2013;94:497-501.

[6] Gennisson J-L, Deffieux T, Fink M, Tanter M. Ultrasound elastography: principles and techniques. Diagn Interv Imaging 2013;94:487-95.

[7] Nakashima K, Shiina T, Sakurai M, Enokido K, Endo T, Tsunoda H, et al. JSUM ultrasound elastography practice guidelines: breast. $J$ Med Ultrason 2013;40:359-91.

[8] Blank MAB, Antaki JF. Breast lesion elastography region of interest selection and quantitative heterogeneity: a systematic review and Meta-Analysis. Ultrasound Med Biol 2017;43:387-97.

[9] Ceyhan Bilgici M, Sağlam D, Delibalta S, Yücel S, Tomak L, Elmalı M. Shear wave velocity of the healthy thyroid gland in children with acoustic radiation force impulse elastography. $J$ Med Ultrason 2018;45:75-80.

[10] Chandramohan A, Therese M, Abhraham D, Paul TV, Mazhuvanchary
PJ. Can ARFI elastography be used to differentiate parathyroid from thyroid lesions? J Endocrinol Invest 2018;41:111-119.

[11] Kudo M, Shiina T, Moriyasu F, Iijima H, Tateishi R, Yada N, et al. JSUM ultrasound elastography practice guidelines: liver. J Med Ultrason 2013;40(4):325-57.

[12] Palabiyik FB, Inci E, Turkay R, Bas D. Evaluation of liver, kidney, and spleen elasticity in healthy newborns and infants using shear wave elastography. J Ultrasound Med 2017;36:2039-2045.

[13] Bayramoglu Z, Caliskan E, Karakas Z, Karaman S, Tugcu D, et al. Diagnostic performances of superb microvascular imaging, shear wave elastography and shape index in pediatric lymph nodes categorization: a comparative study. Br J Radiol 2018;91:20180129.

[14] Tyloch DJ, Tyloch JF, Adamowicz J, Juszczak K, Ostrowski A, Warsiński $\mathrm{P}$, et al. Elastography in prostate gland imaging and prostate cancer detection. Med Ultrason 2018;20:515-523.

[15] Schmalzl J, Fenwick A, Boehm D, Gilbert F. The application of ultrasound elastography in the shoulder. J Shoulder Elbow Surg 2017;26:2236-2246.

[16] Creze M, Nordez A, Soubeyrand M, Rocher L, Maître X, Bellin MF. Shear wave sonoelastography of skeletal muscle: basic principles, biomechanical concepts, clinical applications, and future perspectives. Skeletal Radiol 2018;47:457-71.

[17] Park GY, Kwon DR, Kwon DG. Shear wave sonoelastography in infants with congenital muscular torticollis. Medicine (Baltimore) 2018;97:e9818.

[18] Xiuying Shi, Hong Ai, Juan Wang. Preliminary application of realtime ultrasound tissue elastography in diffuse thyroid diseases. Chinese Journal of ultrasound imaging 2011;20:515-8. [In Chinese]

[19] Yanagisawa O, Niitsu M, Kurihara T, Fukubayashi T. Evaluation of human muscle hardness after dynamic exercise with ultrasound realtime tissue elastography: a feasibility study. Clin Radiol 2011;66:8159.

[20] Zhaoyang Wen, Chunzhi Fan, Lichun An, Jianhong Xu, Hao Chen, Yuexiang Wang. Real time quantitative ultrasound elastography in detecting the difference of elastic modulus of biceps brachii muscle under relaxation and tension. Chinese medical ultrasonic Journal (Electronic Edition) 2011;8:129-34. [In Chinese]

[21] Nakarnura M, Ikezoe T, Kobayashi T, Umeqaki H, Takeno Y, Nishishita S, et al. Acute effects of static stretching on muscle hardness of the medial gastrocnemius muscle belly in humans: an ultrasonic shear-wave elastography study. Ultrasound Med Biol 2014;40(9):1991-7.

[22] Bamber J, Cosgrove D, Dietrich CF, Fromageau J, Bojunga J, Calliada F, et al. EFSUMB guidelines and recommendations on the clinical use of ultrasound elastography. Part 1: Basic principles and technology. Ultraschall Med 2013;34;169-84

[23] Papazoglou S, Rump J, Braun J, Sack I. Shear wave group velocity inversion in MR elastography of human skeletal muscle. Magn Reson Med 2006;56:489-97.

[24] Chino K, Kawakami Y, Takahashi H. Tissue elasticity of in vivo skeletal muscles measured in the transverse and longitudinal planes using shear wave elastography. Clin Physiol Funct Imaging 2017;37:394-399.

[25] Bin Jiang, Xuemei Wang, Yunzhong Wang, Chang Liu. The effect of different planes of sound plane and muscle bundle on the measurement of Young's modulus of SCM muscle was studied by real-time shear wave elastography. Medical imaging technology in China 2014; 30: 269-72. [In Chinese] 